\title{
Improving Access to Psychological Therapies and antidepressant prescribing rates in England:
}

\author{
a longitudinal time-series analysis
}

\begin{abstract}
Background

Antidepressant prescribing rates in England

have been increasing since the 1970s.

The impact of the Improving Access to

Psychological Therapies (IAPT) initiative on

antidepressant prescribing rates is unknown.

\section{Aim}

To investigate the impact of the establishment of IAPT services on antidepressant

prescribing rates in primary care trusts (PCTs) in England.
\end{abstract}

\section{Design and setting}

A longitudinal time-series analysis, using PCTlevel data from 2008 to 2011 set in England.

\section{Method}

A time-series analysis was conducted using PCT-level prescription data, dates of establishment of IAPT services, and covariate data for age, sex, and socioeconomic status. Statistical analysis was carried out using analysis of variance and a random-effect negative binomial model.

\section{Results}

Antidepressant prescribing rates in England increased by $10 \%$ per year during the study period ladjusted rate ratio $=1.10,95 \% \mathrm{Cl}=1.09$ to 1.10). The implementation of IAPT services had no significant effect on antidepressant prescribing ladjusted rate ratio $=0.99,95 \%$ $\mathrm{Cl}=0.99$ to 1.00 )

\section{Conclusion}

Introduction of a large-scale initiative to increase provision of psychological therapies has not curbed the long-term increased prescribing of antidepressants in England.

\section{Keywords}

antidepressive agents; cognitive behavioural therapy; depression; general practice; primary health care.

\section{INTRODUCTION}

Improving Access to Psychological Therapies (IAPT) is a large-scale Department of Health initiative aiming to increase provision of psychological therapies in line with National Institute for Health and Care Excellence (NICE) guidelines for the treatment of depression and anxiety disorders. ${ }^{1}$ Central to IAPT is the provision of evidence-based therapies, particularly cognitive behavioural therapy (CBT), for the treatment of depression and anxiety disorders in a 'stepped care approach. NICE guidelines recommend lowintensity psychological therapy, for example, guided self-help or bibliotherapy, for subthreshold, or mild-to-moderate depression and mild anxiety disorders. High-intensity therapy, for example one-to-one CBT, in combination with antidepressant medication, is recommended for moderate and severe depression and many anxiety disorders, or when there is failure to respond to lowintensity interventions. ${ }^{2}$

IAPT was established in two demonstration sites in $2006-7^{3}$ and then rolled out in waves in 2008-9, 2009-10 and 2010-12. By 2012, it was available in all 151 primary care trusts (PCTs) in England and was treating around $10 \%$ of the population predicted to have anxiety and depression. ${ }^{4}$ PCTs needed to fulfil certain criteria, such as conducting a needs assessment, before being chosen as an IAPT site. The size of the workforce

V Sreeharan, MRCP, MFPH, academic clinical fellow in public health; JT Lee, PhD, research associate in health economics; C Millett, PhD, FFPH, reader in public health; $\mathbf{A}$ Majeed, MD, FRCGP, FFPH, professor of primary care, Department of Primary Care and Public Health, Imperial College, London, Reynolds Building, Charing Cross Campus, London. H Madden, BA PGDip, trainee clinical psychologist, Department of Applied Psychology, Canterbury Christ Church University, Tunbridge Wells.

\section{Address for correspondence}

Vaishnavee Sreeharan, Department of Primary required in each IAPT service was calculated based on the likely prevalence of depression and anxiety in the PCT population (derived from survey data). ${ }^{5}$

Antidepressant prescribing has been increasing in England since the 1970s. ${ }^{6,7}$ The reasons for this are not fully understood, but there is no evidence for increased incidence, prevalence, care-seeking behaviour or GP identification of depression. ${ }^{8}$ One possible explanation is an increase in the proportion of patients receiving long-term treatment with repeat prescriptions. ${ }^{9}$ Despite clear NICE guidance based on current scientific evidence, ${ }^{10}$ there is a divergence of opinion among GPs about the clinical effectiveness of prescribing antidepressants. ${ }^{11,12}$

An economic argument for the establishment of IAPT was based on a cost-benefit analysis that mainly focused on the benefits of increased employment, in terms of the increased revenue gained from returning people to work. ${ }^{13}$ Evaluation of clinical and employment outcomes are central to the IAPT programme, and have shown some positive early results. ' Layard et al's paper also hypothesised the potential savings to the NHS, in reduced costs of secondary care referrals for medically unexplained syndromes and psychiatric conditions, fewer GP visits, and significantly for this study, 'less medication'. ${ }^{13}$

The impact of IAPT on antidepressant prescribing has yet to be established. ${ }^{11}$ The
Care and Public Health, Imperial College, London Reynolds Building, Charing Cross Campus, St Dunstans Road, London, W6 8RP.

E-mail: vaishnaveeddoctors.org.uk Submitted: 27 July 2010; Editor's response: 7 September 2010; final acceptance: 22 October 2010

\section{CBritish Journal of General Practice}

This is the full-length article (published online 27 Aug 2013) of an abridged version published in print. Cite this article as: Br J Gen Pract 2013; DOI: 10.3399/bjgp13X671641 


\section{How this fits in}

Antidepressant prescribing rates in England have been increasing since the 1970s. The cause of this is unknown, but may be due to an increase in long-term prescribing. The Improving Access to Psychological Therapies initiative has not curbed the increase in antidepressant prescribing. Further work is required to explore the reasons for this.

costs of rising antidepressant rates are substantial, and as such, IAPT's impact on these rates should be taken into consideration when calculating the clinical and costeffectiveness of the IAPT programme. There is only one study that investigates the impact of IAPT on healthcare utilisation. ${ }^{14}$ This local before-and-after comparison study showed that after accessing the IAPT service, patients with common mental health problems had increased rates of antidepressant prescriptions, as well as decreased rates of secondary care utilisation and decreased sickness absence, when compared with age and sex matched controls. While the impact of IAPT on healthcare utilisation has yet to be established, there has been some research into the impact of primary care psychological therapy (mainly counselling) on healthcare utilisation. A 2009 Cochrane review concluded that mental health workers working in primary care to deliver psychological therapy caused a significant reduction in GP consultations, prescribing, and secondary care referrals, although the changes were modest, inconsistent, and did not generalise to the wider patient population. ${ }^{15}$

The aim of this study was to investigate the impact of the establishment of IAPT services on antidepressant prescribing rates in PCTs in England.

\section{METHOD}

\section{Sources of data}

Prescription data. The main outcome measure was antidepressant prescription items for PCTs in England for three consecutive time periods: April 2008 to March 2009, April 2009 to March 2010 and April 2010 to March 2011. Antidepressant prescription items included all drugs in the British National Formulary section 4.3 (4.3.1 tricyclic and related antidepressant drugs, 4.3.2 monoamine oxidase inhibitors, 4.3.3 selective serotonin re-uptake inhibitors, and 4.3.4 other antidepressant drugs). ${ }^{16}$ Prescription data were obtained from the NHS Information Centre iView website. ${ }^{17}$ This site provides figures based on information systems at NHS Prescription Services, and includes all NHS prescriptions dispensed in the community. The Prescription Services data are extracted from the ePACT.net system, which allows the NHS Information System to extract data via the NHS Net. There was a high level of data completeness, with missing data for only one PCT, which was excluded.

IAPT service data. The main independent variable was the establishment of an IAPT service. Information was obtained about the date of establishment lin terms of year and quarter) of an IAPT service from the IAPT office at the Department of Health. PCTs were grouped in 'waves' according to when they were established. Wave 1 sites were defined as those with an IAPT service established between April 2008 and March 2009, wave 2 sites between April 2009 and March 2010, and wave 3 sites after April 2010. There are 151 PCTs in England. Data were available for 150 PCTs lincluding 3 PCTs that had not established a service at the time of analysis, and excluding 1 PCT with missing prescription datal.

Covariate data. The main PCT-level covariates were age, sex, and socioeconomic status. In addition, ethnicity was included as a covariate as antidepressant prescribing has been found to be lower in areas with high densities of black or South Asian people. ${ }^{18}$ Two further 'supply-side' covariates were prevalence of depression recorded in general practice lthe prevalence of anxiety was unavailable as it is not recorded in general practice), and GP density.

- GP registered population, age (proportion over 15 years), and sex (proportion of females) were taken from the PCT registered population in 2010 (NHS Information Centre). ${ }^{19}$

- Ethnicity Iproportion of black and ethnic minorityl was taken from the 2009 population estimates by ethnic group (Office for National Statistics). ${ }^{20}$

- Socioeconomic status was taken from the Index of Multiple Deprivation (IMD) levels for 2010 (Department of Communities and Local Government). ${ }^{21}$ IMD data for all PCTs were categorised into five quintiles: the first quintile for the most affluent and the fifth quintile for the most deprived (IMD score range for first quintile: 8.0915.51; second quintile: 15.54-20.34; third quintile: 20.36-26.11; fourth quintile: 26.19-31.36; fifth quintile: 31.79-48.26). 
- The prevalence of depression in each PCT was taken from the Quality and Outcomes Framework (QOF) for 2009-2010.22

- GP density (number of GPs/1000 people) was taken from NHS staff statistics by PCT (NHS Information Centre). ${ }^{23}$

\section{Statistical analysis}

Differences in the characteristics of PCTs in the three waves were tested for using analysis of variance. A time-series analysis was conducted to assess the impact of IAPT by including a continuous variable for the periods after the implementation of IAPT in a PCT. To control for the underlying time trend in antidepressant prescribing rates, the study model included a time variable lequal to 1 for April 2008 to March 2009, 2 for April 2009 to March 2010, and 3 for April 2010 to March 2011). This multivariate model also adjusted for the 'wave' in which the IAPT site was established and sociodemographic characteristics such as age, sex, ethnicity, socioeconomic status,

\section{Table 1. Primary care trust characteristics by wave}

\begin{tabular}{lccccc} 
& & Wave 1 & Wave 2 & Wave 3 & \\
Variable & $\begin{array}{c}\text { All PCTs } \\
\text { (150 PCTs) }\end{array}$ & $\begin{array}{c}\text { 2008-9 } \\
\text { (34 PCTs) }\end{array}$ & $\begin{array}{c}\text { 2009-10 } \\
\text { (77 PCTs) }\end{array}$ & $\begin{array}{c}\text { 2010-11 } \\
\text { (39 PCTs) }\end{array}$ & P-value \\
\hline Proportion of population: aged $>$ 15 & $81.9 \%$ & $82.3 \%$ & $81.9 \%$ & $81.7 \%$ & 0.067 \\
\hline Proportion of population: female & $50.0 \%$ & $50.1 \%$ & $50.0 \%$ & $50.1 \%$ & 0.180 \\
\hline Proportion of population: black and ethnic minority & $13.5 \%$ & $11.7 \%$ & $12.8 \%$ & $16.6 \%$ & 0.004 \\
\hline Prevalence of depression & $7.6 \%$ & $7.7 \%$ & $7.4 \%$ & $7.6 \%$ & 0.370 \\
\hline Number of GPs/10 000 people & 5.6 & 5.7 & 5.6 & 5.3 & $<0.001$ \\
\hline Average IMD score & 23.9 & 23.3 & 24.0 & 24.2 & 0.720 \\
\hline IMD = Index of Multiple Deprivation. PCT = primary care trust. & & & &
\end{tabular}

\section{Table 2. Antidepressant prescribing rate ratios}

\begin{tabular}{lccc} 
Variable & ARR & $\mathbf{9 5 \%} \mathbf{C l}$ & $\boldsymbol{P}$-value \\
\hline Time period, years & 1.10 & 1.09 to 1.10 & $<0.001$ \\
\hline Years after establishment of IAPT & 0.99 & 0.99 to 1.00 & 0.61 \\
\hline Wave 1 & & (reference group) \\
Wave 2 & 1.00 & 0.94 to 1.06 & 0.99 \\
Wave 3 & 0.98 & 0.91 to 1.05 & 0.55 \\
\hline Proportion of population: age $<15$ years & 0.99 & 0.97 to 1.00 & 0.13 \\
\hline Proportion of population: female & 0.95 & 0.92 to 0.98 & 0.001 \\
\hline Proportion of population: ethnic minority & 0.97 & 0.97 to 0.98 & $<0.001$ \\
\hline QOF prevalence of depression, \% & 1.09 & 0.89 to 1.34 & 0.38 \\
\hline Number of GPs per 10 000 population & 1.03 & 0.98 to 1.07 & 0.29 \\
\hline Socioeconomic status (the most affluent as reference group) & & & \\
Second quintile & 1.05 & 0.98 to 1.13 & 0.19 \\
Third quintile & 1.16 & 1.08 to 1.26 & $<0.001$ \\
Fourth quintile & 1.22 & 1.13 to 1.32 & $<0.001$ \\
Fifth quintile (the most deprived) & 1.32 & 1.20 to 1.44 & $<0.001$ \\
\hline ARR = antidepressant prescribing rate ratio. QOF = Quality and Outcomes Framework. & &
\end{tabular}

prevalence of depression, and number of GPs per 10000 people.

A negative binomial model was used to account for the overdispersion of the outcome measure and a random effect specification to account for clustering of repeated measures within PCTs. The rate ratio was estimated by including an offset term, with coefficient equal to 1 , to account for the different population sizes in each PCT. ${ }^{24}$

Multicollinearity for the covariates controlled for was tested for in the analysis. The multicollinearity diagnostics (VIF = Variance Inflation Factor) were all less than 5, indicating that the assumption of reasonable independence among predictor variables was met. All the analyses were performed using Stata (version 10).

\section{RESULTS}

Descriptive statistics for PCT characteristics by wave are shown in Table 1. There were data for 150 PCTs in total. There was a statistically significant difference in the ethnic group composition and GP density between the three waves, with wave 1 sites having the lowest proportion of black and ethnic minority residents and the highest number of GPs/10 000 people. There was no statistically significant difference in the other covariates between the three waves. The significant difference in certain covariates between the three waves highlights the importance of controlling for these in the regression model.

Results for the regression analysis can be found in Table 2. From 2008 to 2011, there was a mean increase in antidepressant prescription rate of $10 \%$ per year ladjusted rate ratio $=1.10,95 \% \mathrm{Cl}=1.09$ to 1.10 ) . There was no significant effect of IAPT on antidepressant prescriptions ladjusted rate ratio $=0.99,95 \% \mathrm{Cl}=0.99$ to 1.00 ).

To test whether IAPT had a differential impact on prescribing rates depending on the wave of implementation, an interaction term was included between wave and IAPT variables. The results suggested that IAPT's impact on antidepressant prescribing; however, did not vary by the different waves (P>0.05).

Results from the covariates demonstrated that sex, ethnicity, and socioeconomic status were associated with antidepressant use (Table 2). A $1 \%$ increase in the female population was associated with a $5 \%$ decrease in the prescription rate ratio. A $1 \%$ increase in the ethnic minority population was associated with a 3\% decrease in the prescription rate ratio. Increasing socioeconomic deprivation had particularly 
marked associations with antidepressant use. The more deprived areas had higher antidepressant prescribing rates: compared with the most affluent areas, the rate ratio for the most deprived was 1.32 195\% $\mathrm{Cl}=1.20$ to 1.44$)$.

\section{DISCUSSION}

\section{Summary}

Increasing antidepressant prescribing rates for PCTs in England were found, with a rise of $10 \%$ per year over the study period 2008-2011. The implementation of IAPT had no significant impact on these increasing rates.

\section{Strengths and limitations}

This is the first national study to assess the association between establishment of an IAPT service and antidepressant prescribing rates, using a reliable source of national prescription data. Such an ecological study may be useful for 'hypothesis generating' so that the results can be investigated in further observational studies using individuallevel data. The study controlled for the time trend and several important covariates in the analysis, including demographic characteristics of PCT populations.

Interpretation of these findings are limited by the 'ecological fallacy' that results when making causal inferences from group data to individual behaviours. ${ }^{25}$ For example, in the current study it cannot be concluded that individuals who were receiving antidepressants in each PCT were those who were in contact with the IAPT service. In addition, although the ecological study adjusted for many confounding factors, it did not account for comorbidity and the size and access rates of each IAPT service, as these data were unavailable. There is a wide variation in the number of therapists in each service, and hence, a variation in the number of clients able to access IAPT. There are also certain limitations of routinely collected prescribing data. They only provide a narrow range of information (what drugs are prescribed and their cost), and cannot be linked to demographic or clinical data on patients. ${ }^{26}$ Furthermore, they do not provide information about the indications for which the drug was prescribed, and so it was not possible to ascertain what proportion of antidepressants prescribed during the study period were prescribed for depression.

Another limitation is the short timescale (2008-2011) used in the study. It may take more time for IAPT to become more widespread and to exert its full impact on antidepressant prescribing rates.

\section{Comparison with existing literature}

The rise in antidepressant prescribing rates over time is consistent with several existing studies.6.7 Previous studies have found few reasons to explain this general rise other than an increase in the proportion of patients receiving long-term treatment with repeat prescriptions. ${ }^{8,9}$ The lack of impact of IAPT on antidepressant prescribing at a population level is supported by several studies. For example, a 2009 Cochrane review concluded that any reduction in prescribing that occurred when individuals had access to psychological therapy did not generalise to the wider practice population/community. ${ }^{15}$ This finding is corroborated by other primary studies. ${ }^{27,28}$ A recent economic evaluation of an IAPT demonstration site found that IAPT provided a service that was probably costeffective' within the usual NICE threshold range of $€ 20000-€ 30000$, although with considerable uncertainty surrounding the costs and outcome differences. ${ }^{29}$ This evaluation considered the lack of inclusion of medication costs as a study limitation. ${ }^{29}$

\section{Implications for research and practice}

These findings will be useful additional information for future economic evaluations of IAPT, and as such, will be of interest to those commissioning services, in particular, GPs in England from April 2013.

The interpretation of antidepressant prescribing rates is complex. Several recent factors may impact on diagnosis and management of depression. For example, QOF incentives following a diagnosis of depression may either increase rates or alternatively discourage GPs from applying a 'label' because of the workload involved. In addition, waiting times for IAPT may necessitate intermediate action by GPs, such as prescribing antidepressants. As these results suggest that IAPT has not curbed the rise in antidepressant prescribing, it is important to re-explore factors that influence this prescribing. This may involve qualitative studies investigating the influences of GPs' prescribing patterns, particularly with regards to attitudes towards IAPT, and the role of medication in the treatment of depression and anxiety. Interviewing patients would also be of worth.

It would be useful to repeat the current study in a few years to ascertain the longerterm impact of IAPT on antidepressant prescription trends. It may also be informative to include changes in anxiolytic prescription rates, as IAPT aims to treat both depression and anxiety disorders. 


\section{REFERENCES}

1. Clark DM. Implementing NICE guidelines for the psychological treatment of depression and anxiety disorders: the IAPT experience. Int Rev Psychiatry 2011; 23(4): 318-327.

2. National Institute for Health and Clinical Excellence. Depression: the treatment and management of depression in adults (updated edition). CG90. London: NICE, 2009

3. Clark DM, Layard R, Smithies R, et al. Improving access to psychological therapy: Initial evaluation of two UK demonstration sites. Behav Res Ther 2009; 47(11): 910-920.

4. Improving Access to Psychological Therapies. Performance (KPI omnibus collection). http://uww.iapt.nhs.uk/data/current-performance/ laccessed 18 Jul 2013).

5. Care Services Improvement Partnership, National Institute for Mental Health in England. Improving Access to Psychological Therapies (IAPT) commissioning toolkit. http://uww.icn.csip.org.uk/_library/IAPT_ commissioning_toolkit.pdf (accessed 18 Jul 2013).

6. Middleton N, Gunnell D, Whitley E, et al. Secular trends in antidepressant prescribing in the UK, 1975-1998. J Public Health Med 2001; 23(4): 262-267.

7. Ilyas S, Moncrieff J. Trends in prescriptions and costs of drugs for mental disorders in England, 1998-2010. Br J Psychiatry 2012; 200(5): 393-398.

8. Munoz-Arroyo R, Sutton M, Morrison J. Exploring potential explanations for the increase in antidepressant prescribing in Scotland using secondary analyses of routine data. Br J Gen Pract 2006; 56(527): 423-428.

9. Moore M, Yuen HM, Dunn N, et al. Explaining the rise in antidepressant prescribing: a descriptive study using the general practice research database. BMJ 2009; 339: b3999

10. Fournier JC, DeRubeis RJ, Hollon SD, et al. Antidepressant drug effects and depression severity: a patient-level meta-analysis. JAMA 2010; 303(1): 47-53.

11. Middleton H, Moncrieff J. 'They won't do any harm and might do some good': time to think again on the use of antidepressants? Br J Gen Pract 2011; 61(582): 47-49.

12. Anderson IM, Haddad PM. Prescribing antidepressants for depression: time to be dimensional and inclusive. Br J Gen Pract 2011; 61(582): 50-52.

13. Layard R, Clark D, Knapp M, et al. Cost-benefit analysis of psychological therapy. London: Centre for Economic Performance, London School of Economics and Political Science, 2007

14. de Lusignan S, Chan T, Parry G, et al. Referral to a new psychological therapy service is associated with reduced utilisation of healthcare and sickness absence by people with common mental health problems: a before and after comparison. J Epidemiol Community Health 2012; 66(6): e10.
15. Harkness EF, Bower PJ. On-site mental health workers delivering psychological therapy and psychosocial interventions to patients in primary care: effects on the professional practice of primary care providers. Cochrane Database Syst Rev 2009 Jan 21;(1):CD000532.

16. Joint Formulary Committee. British National Formulary 62. London: BMJ Group and Pharmaceutical Press, 2011.

17. NHS The Information Centre. NHS iView. PCT Prescribing. https://iview.ic.nhs. uk/DomainInfo/PCTPrescribing laccessed 6 Aug 2013).

18. Walters P, Ashworth M, Tylee A. Ethnic density, physical illness, social deprivation and antidepressant prescribing in primary care: ecological study. Br J Psychiatry 2008; 193(3): 235-239

19. Health \& Social Care Information Care Information Centre. Attribution Data Set GP-Registered Populations - 2010. http://www.hscic.gov.uk/searchcatalog ue? productid $=4285 \& q=$ title $\% 3 a \% 22$ Attribution+data+set+GP-registered+popu lations\%22\&sort=Relevance \&size=10\&page=1\#top (accessed 6 Aug 2013)

20. Office for National Statistics. Population estimates by ethnic group (experimental), mid-2009. http://www.ons.gov.uk/ons/publications/ re-reference-tables.html?edition=tcm\%3A77-50029 (accessed 18 Jul 2013),

21. Department for Communities and Local Government. English indices of deprivation 2010. http://uww.communities.gov.uk/publications/corporate/ statistics/indices2010 (accessed 18 Jul 2013).

22. Health \& Social Care Information Care Information Centre. Quality and Outcomes Framework - 2009-10. http://www.hscic.gov.uk/catalogue/ PUB04431 (accessed 6 Aug 2013).

23. Health \& Social Care Information Centre. http://umw.hsic.gov.uk/searchcatalogue? productid=10382\&topics=1\%2fWork-force $\% 2 f$ Staff=numbers \&sort=Rel evance \&size=10\&page= 1 \#top (accessed 6 Aug 2013)

24. Cummings $P$. Population and primary health care characteristics and coronary heart disease mortality. JAMA 2011; 305(8): 778

25. Schwartz S. The fallacy of the ecological fallacy: the potential misuse of a concept and the consequences. Am J Public Health 1994; 84(5): 819-824.

26. Majeed A, Evans N, Head P. What can PACT tell us about prescribing in general practice? BMJ 1997; 315(7121): 1515-1519.

27. Baker R, Allen H, Gibson S, et al. Evaluation of a primary care counselling service in Dorset. Br J Gen Pract 1998; 48(428): 1049-1053.

28. Simpson S, Corney R, Fitzgerald P. Counselling provision, prescribing and referral rates in a general practice setting. Int J Psychiatry Clin Pract 2003 8(4): 115-119.

29. Mukuria C, Brazier J, Barkham M, et al. Cost-effectiveness of an improving access to psychological therapies service. Br J Psychiatry 2013; 202(3): 220227. 